\title{
Interactive Multimedia Explanation for Equipment Maintenance and Repair
}

\author{
Kathleen McKeown and Steven Feiner
}

\author{
Department of Computer Science \\ 450 Computer Science Building \\ Columbia University \\ New York, N.Y. 10027
}

\section{Objectives}

We are developing COMET, an interactive system that generates multimedia explanations of how to operate, maintain, and repair equipment. Our research stresses the dynamic generation of the content and form of all material presented, addressing issues in the generation of text and graphics, and in coordinating text and graphics in an integrated presentation.

COMET contains a static knowledge base describing objects and plans for maintenance and repair, and a dynamic knowledge source for diagnosing failures. Explanations are produced using a content planner that determines what information should be communicated, a media coordinator that determines which information should be realized in graphics and which in text, and separate text and graphics generators. The graphics and text for a single explanation are laid out on the screen by a media layout component. A menu interface allows users to request explanations of specific procedures or to specify failure symptoms that will invoke a diagnostic component. The diagnostic component can ask the user to carry out procedures that COMET will explain if requested. In contrast to hypermedia systems that present previously authored material, COMET has underlying models of the user and context that allow each aspect of the explanation generated to be based on the current situation.

Our focus in the text generation component has been on the development of the Functional Unification Formalism (FUF) for non-syntactic tasks, of a large syntactic grammar in FUF, of lexical choice in FUF using constraints from underlying knowledge sources and from past discourse, and of models of constraints on several classes of word choice. Important results in the graphics component include the knowledge-based design of 3D illustrations, a design-grid-based methodology for display layout, and the development of a novel graphical history interface that allows users to review previous sessions.
Finally, we have had significant results in the development of our media coordinator which, unlike other systems, features a common description language that allows a finegrained division of information between text and graphics. The media coordinator maps information to media specific resources, and allows information expressed in one media to influence realization in the other. This allows for tight integration and coordination between different media.

\section{Recent Accomplishments}

- Integrated the different systems of which COMET is comprised to produce a running testbed that creates multimedia explanations on-the-fly, in response to user requests. Our testbed system generates and lays out a full display of text and graphics in 15-25 seconds.

- Incorporated user model constraints on content generation, to avoid presenting information that has been presented previously.

- Extended the support for using FUF in vocabulary choice, adding the ability to access the knowledge base from the lexicon to influence the choice of vocabulary.

- Added the ability to use previous discourse to influence the choice of vocabulary.

- Began implementing support for coordinating picture and text breaks.

- Began implementing support for cross-references between text and graphics.

- Extended the ability of the graphics generator to represent actions by means of metaobject arrows.

- Began the preliminary design of facilities for user exploration of the generated explanation, including the ability to examine depicted objects from different viewpoints than those chosen by the system. 